\title{
Study of a Mini-Actuator with Permanent Magnets
}

\author{
Camelia PETRESCU, Radu OLARU \\ Faculty of Electrical Engineering, Gh. Asachi Technical University of Iasi \\ Bd. Dimitrie Mangeron nr.53, RO-700050 Iaşi, Romania \\ campet@ee.tuiasi.ro
}

\begin{abstract}
The paper presents an analytical method for the determination of the magnetic force produced by a mini actuator with permanent magnets. The results are compared with those obtained by performing a numerical field analysis with COMSOL Multiphysics, showing a very good agreement. The study reveals that the actuator has two equilibrium points, one of which is stable and the other one unstable.
\end{abstract}

Index Terms - actuators, analytical and numerical methods, magnetic fields

\section{INTRODUCTION}

Actuators are used to transmit forces (or pressures, or torques) and often represent an end component in an automation chain (for example in the control of pneumatic valves). Technical literature presents numerous types of actuators, mostly using the magnetic energy produced by coils or permanent magnets. Some examples are [1 - 4]. Much of the research involves the application of highenergy rare-earth permanent magnets, which have facilitated the development of new magnetic actuators with improved performance [5].

Linear moving magnet actuators have increasing practical importance in generating motion. In some medical applications, moving magnet actuators can generate the motion needed for realistic modeling of human body dynamics. The Bose BioDynamic Corp. [6] uses several types of moving magnet actuators to insert a prototype disc through the wringer. Another Bose application is an electromagnetic suspension system based on moving magnet linear actuators. A miniature short stroke linear actuator with mobile magnets for robotic applications is described in [7].

In the design of an actuator the characteristic force versus displacement of the mobile part and command current must be known, thus enabling a choice of the geometric and material properties for enhanced performance.

In our previous papers, analytical methods for the determination of forces exerted by actuators, based on ferro fluids and having a mobile non magnetic disc were established [8-9].

The present paper presents a study of a moving magnet actuator with three permanent magnets and a command coil. The magnetic field and the force acting on the mobile magnet are determined both analytically and numerically using the software COMSOL Multiphysics 3.5, based on the finite element method. Finally, the actuator characteristic driving force versus magnet displacement is established.

\section{ACTUATOR CONFIGURATION AND ANALYTICAL FORCE DETERMINATION}

The physical model of the actuator is presented in Figure 1. The coil, with two identical sections that are connected in series or supplied with equal currents in opposite directions, carries the command current $i$. Two identical fixed exterior magnets are used to ensure a stable position of the central mobile magnet at the middle of the device when $i=0$. In the case of an "on - off" actuator, DC currents are used, while, if the device is used as a micro - pump, $i$ is a very low frequency sinusoidal current.

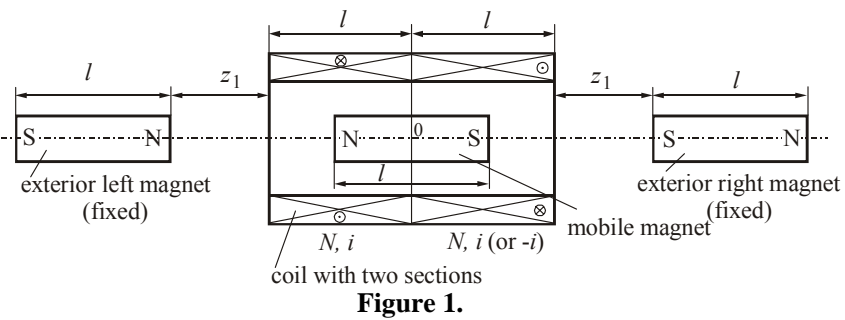

The whole device has axial symmetry and can be analyzed in the $(r, z)$ plane.

The central magnet has the radius $r_{m 1}$ and the uniform remanent magnetic flux density $B_{r 1}$, while the outer magnets have the radii $r_{m 2}$ and the uniform magnetic flux density $B_{r 2}$. Each section of the multilayered coil has $N$ turns and the interior and exterior radii $r_{1}$ and $r_{2}$, respectively. All magnets and coil sections have the same length $l$.

The first step in calculating the force acting on the central magnet is to determine the magnetic field produced by the multilayer coil. Considering a layer of thickness $\mathrm{d} r$ containing $\frac{N l \mathrm{~d} r}{l\left(r_{2}-r_{1}\right)}$ wires, the infinitesimal magnetic field it produces at a point $P(z)$ (Figure 2) is:

$$
\mathrm{d} H_{1}=\frac{N i \mathrm{~d} r}{\left(r_{2}-r_{1}\right) 2 l}\left(\frac{z}{\sqrt{z^{2}+r^{2}}}-\frac{z-l}{\sqrt{(z-l)^{2}+r^{2}}}\right)
$$

Thus the total magnetic field produced by the left section of the coil is:

$$
\begin{array}{r}
H_{1}(z)=\int_{r_{1}}^{r_{2}} \mathrm{~d} H_{1}=\frac{N i}{2 l\left(r_{2}-r_{1}\right)}\left(z \ln \frac{r_{2}+\sqrt{r_{2}^{2}+z^{2}}}{r_{1}+\sqrt{r_{1}^{2}+z^{2}}}-\right. \\
\left.-(z-l) \ln \frac{r_{2}+\sqrt{r_{2}^{2}+(z-l)^{2}}}{r_{1}+\sqrt{r_{1}^{2}+(z-l)^{2}}}\right)
\end{array}
$$




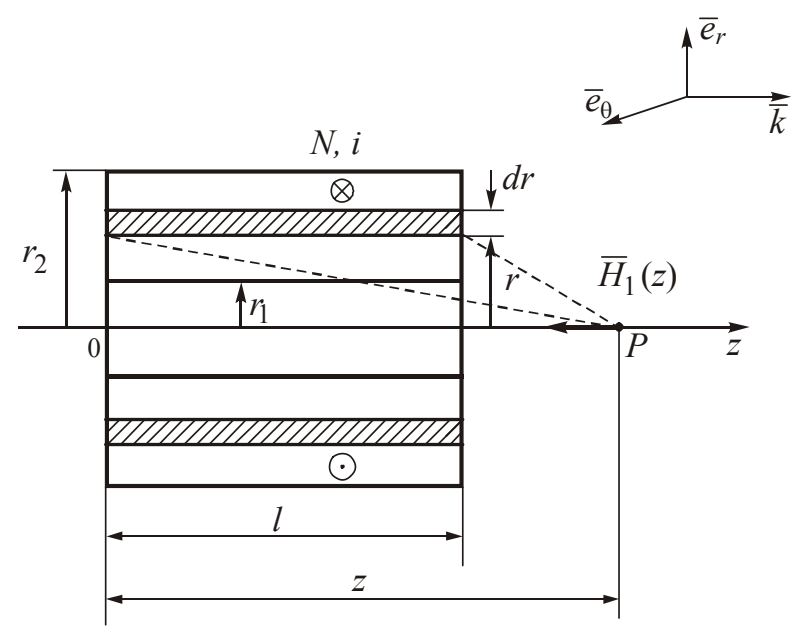

Figure 2.

In the same way the magnetic field produced by the right section of the coil in the same point is:

$$
\begin{aligned}
H_{2}(z)=\int_{r_{1}}^{r_{2}} \mathrm{~d} H_{2}= & \frac{N i}{2 l\left(r_{2}-r_{1}\right)}\left((z-l) \ln \frac{r_{2}+\sqrt{r_{2}^{2}+(z-l)^{2}}}{r_{1}+\sqrt{r_{1}^{2}+(z-l)^{2}}}\right. \\
& \left.-(z-2 l) \ln \frac{r_{2}+\sqrt{r_{2}^{2}+(z-2 l)^{2}}}{r_{1}+\sqrt{r_{1}^{2}+(z-2 l)^{2}}}\right)
\end{aligned}
$$

If the coils are in series, the total field is $H(z)=H_{1}(z)+H_{2}(z)$, while if the currents in the two sections have opposite directions, the magnetic field intensity is $H(z)=H_{1}(z)-H_{2}(z)$.

Considering that the inner magnet has a displacement $x$ from the central position, the force acting in axial direction on the central magnet may be calculated with:

$$
F_{z}=\left.\frac{\partial W_{m}}{\partial x}\right|_{i=\text { const }}
$$

where $W_{m}$ is the magnetic energy of the system.

The inner permanent magnet can be substituted by a cylindrical thin sheet of radius $r_{m 1}$ carrying the amperian surface current density [10-11]

$\boldsymbol{J}_{A s}=-M_{p 1} \boldsymbol{e}_{\theta}=-\frac{B_{r 1}}{\mu_{0}} \boldsymbol{e}_{\theta}$.

Considering the case $r_{m 1}<<r_{1}$, the magnetic flux produced by the current $i$ in a transversal section of the inner permanent magnet may be very well approximated by $\Phi(z)=\mu_{0} H(z) \pi r_{m 1}^{2}$. Denoting by $W_{1}=W_{m}(x)$ the fraction of the total magnetic energy of the device that depends on the displacement $x$, the energy corresponding to an infinitely small section $\mathrm{d} z$ of the inner magnet is:

$\mathrm{d} W_{1}=\mu_{0} H(z) \pi r_{m 1}^{2} J_{A s} \mathrm{~d} z$.

The total magnetic energy, the term depending on $x$, has the expression:

$$
W_{1}(x)=B_{r 1} \pi r_{m 1}^{2} \int_{\frac{l}{2}+x}^{\frac{3 l}{2}+x}\left[H_{1}(z) \pm H_{2}(z)\right] \mathrm{d} z,
$$

the plus sign in relation (7) corresponding to the case when the currents in the two coil sections have the same direction, and the minus sign for opposite current directions.

The distance $z_{1}$ between the exterior magnets and the two coils is larger than the length $l\left(z_{1} \approx 3 l\right.$ in the studied case), so that the influence of these magnets in determining the overall force can be neglected, as the numerical simulations presented in the following paragraph demonstrate.

\section{NUMERICAL RESULTS}

The integral in relation (7) cannot be calculated analytically, so that a numerical quadrature and differentiation had to be performed using the MATLAB functions quad and diff, in order to evaluate $W_{1}(x)$ and $F_{z}$ with relations (7) and (4). At the same time the device was analyzed using COMSOL Multiphysics 3.5.a, a software designed to solve single or coupled physics problems described by partial differential equations (PDEs) using the finite element method (FEM).

In this case the equation solved by COMSOL is: $\nabla \times\left(\mu^{-1}\left(\nabla \times \boldsymbol{A}-\boldsymbol{B}_{\boldsymbol{r}}\right)\right)=J_{\theta}^{e} \boldsymbol{e}_{\theta}$

where $\boldsymbol{A}=A_{\theta} \boldsymbol{e}_{\theta}$ is the magnetic vector potential and $J_{\theta}^{e}$ is the externally generated current density, with $J_{\theta}^{e}= \pm \frac{N i}{\left(r_{2}-r_{1}\right) l}$ in the coil.

Successive tests were first carried out in order to choose the adequate settings for COMSOL. The analyzed domain in the $(r, z)$ plane was a rectangle extending in the $r$ and $z$ directions to the maximum values $r_{\max }=4 \cdot r_{2}=24 \mathrm{~mm}$, $z_{\max }=16 \cdot l=8 \cdot$ coil length $=96 \mathrm{~mm}$.

The mesh for the FEM analysis contained approximatively 15000 elements, the maximum element size in the central magnet and inside the coil being $0.1 \mathrm{~mm}$. Second order Lagrange shape functions were used. COMSOL Multiphysics includes several system solvers, the Geometric multi-grid being the best suited for this case study. This is an iterative solver that performs well for degrees of freedom (DOFs $=$ number of nodes) larger than 100000, [10], (in this example DOF $\in(350000,650000)$ ). The adaptive mesh refinement option was used, which means that the solver computes the solution for an initial and a number of subsequently refined meshes (imposed) until an error condition is satisfied.

The force $F_{z}$ is calculated in COMSOL by integrating the $z$ component of Maxwell's magnetic tensor on the central magnet surface [10]:

$\boldsymbol{F}_{z}=\iint_{S_{\text {magn }}}\left[(\boldsymbol{n} \cdot \boldsymbol{H}) \cdot \boldsymbol{B}^{\boldsymbol{T}}-\frac{1}{2} \boldsymbol{n} \cdot(\boldsymbol{H} \cdot \boldsymbol{B})\right] \mathrm{d} A$

where $\boldsymbol{n}$ is the outward normal from the magnet.

The numerical values used in simulations were: $r_{1}=3.6 \mathrm{~mm}, \quad r_{2}=6 \mathrm{~mm}, \quad r_{m 1}=1.5 \mathrm{~mm}, \quad r_{m 2}=1 \mathrm{~mm}, \quad B_{r 1}=0.43 \mathrm{~T}$, $B_{r 2}=0.25 \mathrm{~T}, l=6 \mathrm{~mm}, z_{1}=17 \mathrm{~mm}, i=0.1 \mathrm{~A}, \mathrm{~N}=500$ wires. The step used in the numerical differentiation with MATLAB was $10^{-5} \mathrm{~mm}(l / 600)$.

The system without exterior magnets was firstly analyzed using the two methods in order to appreciate the accuracy.

Figure 3 presents the plot force acting in the $z$ direction, $F_{z}$, versus central magnet displacement, $x$, in the case of additive magnetic fields. Figure 4 illustrates the dependence $F_{z}(x)$ for the case of differential magnetic fields (currents in opposite directions in the coil sections).

The following observations can be made:

- the results based on the analytical solution for the magnetic field and those obtained with COMSOL Multiphysics are in very good agreement;

- $\quad$ in the case of additive magnetic fields the inner magnet is stable $\left(F_{z}=0\right)$ in the central position $(x=0)$, while 
for differential magnetic fields two equilibrium positions are apparently obtained $-F_{z}=0$ gives two equal values for $|x|$;

a force $F_{z}>0$ acts in the positive $z$ direction leading to an increase in $x$, while a force $F_{z}<0$ moves the magnet in the negative $z$ direction (to the left in Figure 1);

the last two observations show that for the case of differential magnetic fields (Figure 4) there is in fact only one stable equilibrium point for the inner magnet, the left one, $x<0, F_{z}=0$;

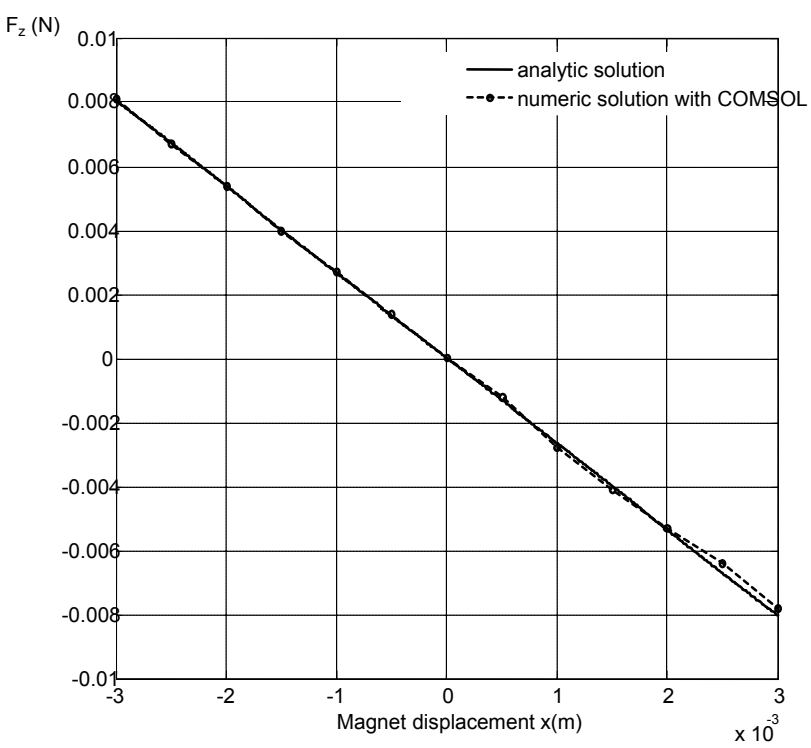

Figure 3.

- the maximum force, in modulus, is obtained for the magnet in the central position $(x=0)$, in the case of currents in opposite directions, $F_{z}=-0.0182 \mathrm{~N}$;

- for the analyzed system, the equilibrium position of the interior magnet corresponds to its middle point placed inside the coil at a distance $x=-4.4 \mathrm{~mm}=-0.732 \cdot l$.

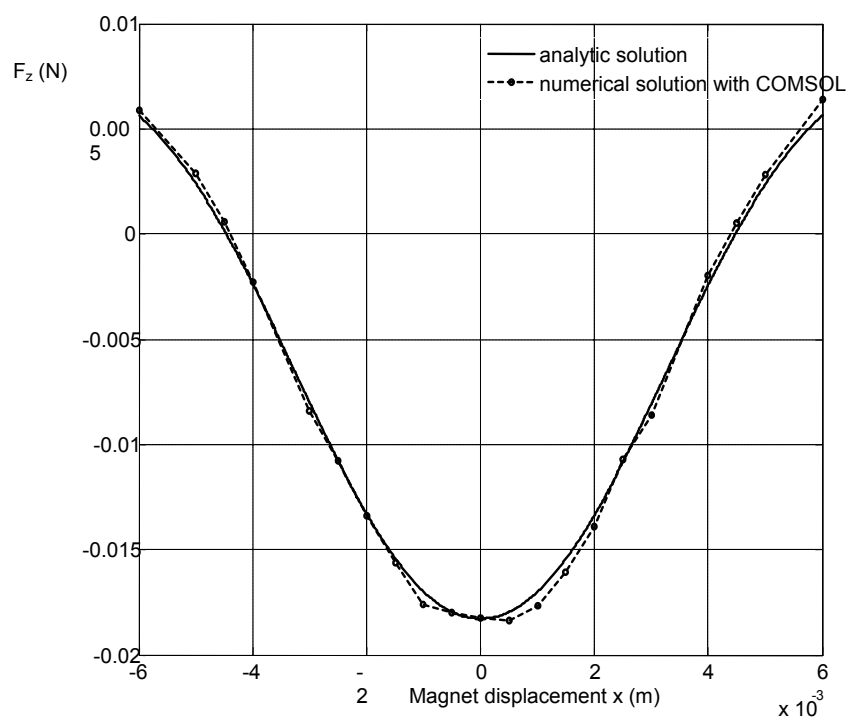

Figure 4.

The next step was to calculate the force $F_{z}(x)$ with COMSOL for the real actuator, when the two exterior magnets are present (a case for which an analytical solution cannot be obtained). The settings for COMSOL Multiphysics were the same since the accuracy obtained in the first studied case proved very good.
Figure 5 presents the plot $F_{z}(x)$ for the actuator in Figure 1 with the parameters having the same numerical values as before and currents in opposite directions. The range of values for the displacement $x$ was extended. As it may be seen, the values of $F_{z}$ for $x \in[-l, l]$ are close to those obtained in the absence of the fixed magnets. This can be explained by the fact that the distance $z_{1}$ is large as compared to $l\left(z_{1} \approx 3 l\right)$ and the principal source of magnetic field is the coil. The same remarks as before can be made concerning the equilibrium positions of the central magnet $\left(F_{z}=0\right)$ : one stable, to the left, and one unstable, symmetric position, to the right. The plot also shows the effect of the repelling force exerted by the fixed magnets: $F_{z}>0, x<-2 l$ and $F_{z}<0, x>2 l$.

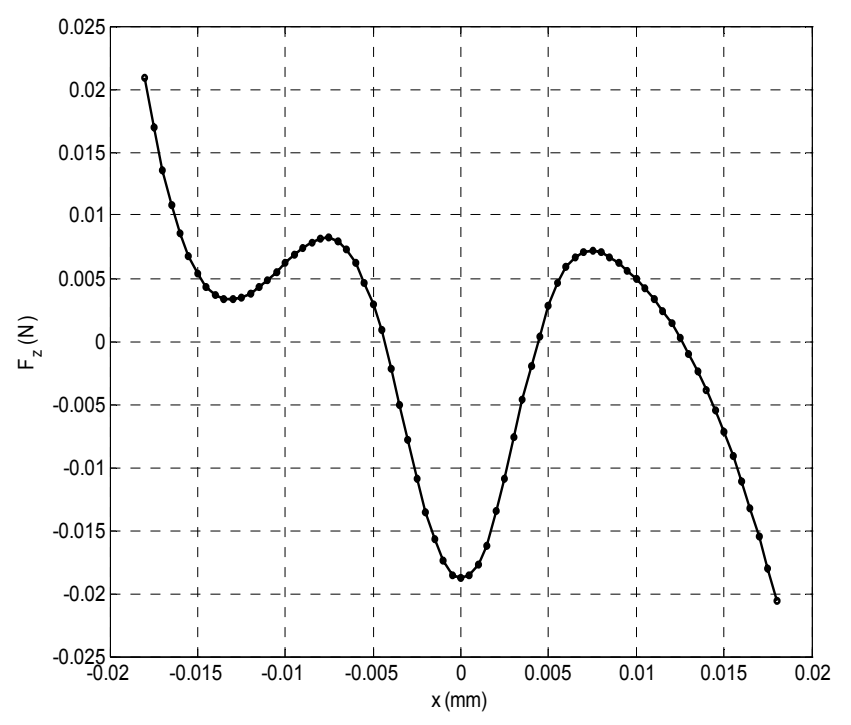

Figure 5.

Figure 6 presents the magnetic field lines for the actuator in Figure 1 with the mobile magnet in central position and differential magnetic fields.

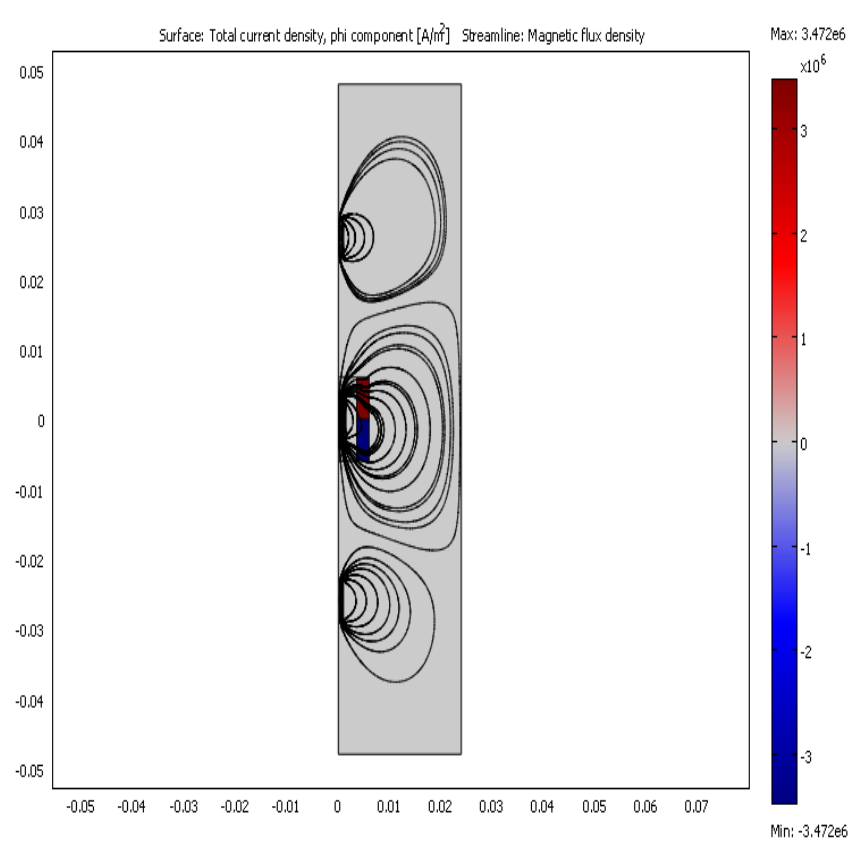

Figure 6. 
This analysis shows the possibility to use the device as a seal for a micro valve, for example, if DC currents in opposite directions are fed into the two sections of the coil. The on-off positions are obtained in this case by switching the current direction in one coil, or in both coils at the same time.

\section{CONCLUSION}

The analysis of the mini - actuator with permanent magnets and a coil with two sections carrying DC currents in opposite directions is focused on the determination of the force acting on the mobile central magnet. The exact solution derived for the magnetic field enables a semi-analytic determination of this force based on magnetic field energy evaluation and on the second generalized magnetic force theorem. The numerical example shows a very good agreement between the results obtained with this method and those obtained using the software COMSOL Multiphysics based on the finite element method.

The study also reveals the existence of a stable and an unstable steady state position of the mobile part of the actuator.

Thus some concluding remarks concerning the reasons for the analysis undertaken in this paper can be made:

- the comparative analysis between the results obtained using the analytical method and the ones obtained using COMSOL is necessary in establishing the settings for the numerical analysis with COMSOL Multiphysics (dimensions of the analyzed domain, number of nodes, shape functions, solver type, etc.);

- the exact analytical formula derived in the paper is valid for the device without exterior magnets, while in the case of the real actuator (with exterior magnets) the analysis is made only with COMSOL;

- the fact that the analysis reveals the existence of two equilibrium positions for the mobile magnet is of theoretical importance since only the stable equilibrium point can be attained experimentally;

- the results obtained from this analysis serve as basis for the experimental study of the real actuator (in progress).

Future studies are intended to outline the influence of the actuator geometrical and magnetic parameters in order to optimize its performance.

\section{REFERENCES}

[1] Z. Q. Zhu, X. Chen, D. Howe and S. Iwasaki, "Electromagnetic modeling of a novel linear oscillating actuator", IEEE Trans. Magnetics, vol. 44, pp. 3855-3858, Nov. 2008

[2] H.S. Lee, Ch. Cho, "Magnetoelastic characteristics of a multilayered magneto strictive thin film with polyimide substrate for microactuator", IEEE Trans. Magnetics, vol. 44, pp.4049-4052, Nov. 2008.

[3] M. Marcovic, M. Jufer and Y. Perriard, "Analytical force determination in an electromagnetic actuator", IEEE Trans. Magnetics, vol. 44, pp.2181-2185, Sept. 2008

[4] K. Hirato, T. Yamamoto, T. Yamaguchi, Y. Kawase and Y. Hasegawa, "Dynamic analysis method of two dimensional linear oscillatory actuator employing finite element method", IEEE Trans. Magnetics, vol. 43, pp.1441-1444, April. 2007

[5] D. Howe, "Magnetic actuators, Sensors and Actuators", vol. 81, pp.268-274, 2000

[a] http://www.motionsystemdesign.com.

[b] H. Lu, J. Zhu, Z. Lin, Y. Guo, "A miniature short stroke linear actuator-Design and analysis", IEEE Transactions on magnetics, Vol. 44, No. 4, 2008, pp. 497-504

[6] R. Olaru, Camelia Petrescu, "Simplified approach for calculating the force of a ferofluidic actuator", Rev. Roum. Des Sciences Techniques, S. Electrot. et Energ., vol. 53, pp.435-443, 2008

[7] R. Olaru, Camelia Petrescu, "Modeling of a ferrofluid actuator with non-magnetic disc", European Magnetic Sensors\&Actuators Conference, EMSA 2008, 30 June-2 July 2008, Caen, France

[8] G. F. Miner. "Lines and Electromagnetic Fields for Engineers". Oxford University Press, 1996

[9] M. Sadiku. "Elements of Electromagnetics". Oxford University Press, 1995

[10] "COMSOL Multiphysics", User's guide, AC/DC Module - User's guide 